\title{
DEVELOPMENT AND CHARACTERIZATION OF CHITOSAN-GRAFTED POLYCAPROLACTONE/POLY (3-HYDROXYBUTYRATE-CO-3-HYDROXYHEXANOATE) FIBER BLENDS FOR TISSUE ENGINEERING APPLICATIONS
}

\author{
A.M. DIEZ-PASCUAL \\ Analytical Chemistry, Physical Chemistry and Chemical Engineering Department, Alcalá University, Spain.
}

\begin{abstract}
Polyhydroxyalkanoates (PHAs) are a family of biodegradable and biocompatible polyesters that have recently attracted much industrial attention. The most representative PHA is poly(3-hydroxybutyrate) (PHB), though it presents several shortcomings such as brittleness and poor impact resistance. 3-hydroxyhexanoate units can be incorporated in PHB to obtain poly(3-hydroxybutyrate-co-3-hydroxyhexanoate) (PHBHHx), a copolymer with improved mechanical properties, processability and biodegradability, more suitable for biomedical applications. In this study, chitosan-grafted polycaprolactone (CS-gPCL)/PHBHHx fiber blends in different compositions were developed by wet electrospinning, and their morphology, biodegradability, mechanical and tribological properties were investigated. A direct correlation was found between the wear rate and the mechanical properties, pointing that fiber breakage is the mechanism responsible for both the abrasive wear and yield. The interactions between the components led to a synergistic effect on tensile and tribological properties at a blend composition of 70/30, resulting in an optimum combination of maximum stiffness, strength, ductility and toughness and minimum coefficient of friction and wear rate, ascribed to the lower porosity and higher crystallinity of this sample. Further, it exhibits the slowest degradation rate. These fiber blends are ideal candidates as scaffolds for tissue engineering applications.
\end{abstract}

Keywords: chitosan, electrospinning, fiber blend, polyhydroxyalkanoates, polycaprolactone, tribological properties, tissue engineering.

\section{INTRODUCTION}

Polyhydroxyalkanoates (PHAs) are a class of biodegradable polyhydroxyesters of 3-, 4-, 5-, and 6 hydroxyalkanoic acids produced by bacteria under nutrient limiting conditions with excess carbon that have recently attracted considerable interest for a variety of applications ranging from medicine, tissue engineering and agriculture to nanocomposites and packaging [1]. The most representative member of this family is poly(3-hydroxybutyrate) (PHB), a biocompatible thermoplastic polyester with good oxygen permeability and good UV resistance, and performance close to that of synthetic polypropylene. Nonetheless, its use has been restricted due to weaknesses such as brittleness and poor impact resistance, relatively high water vapour permeability and low degradation temperature [2]. To overcome these problems, the hydroxybutyrate units can be copolymerized with other monomers like 3-hydroxyhexanoate units, leading to poly(3-hydroxybutyrate-co-3-hydroxy-hexanoate) (PHBHHx), a copolymer with better mechanical properties, processability and biodegradability [3]. In order to spread its range of applications, new strategies should be tested like the incorporation of fillers, surface modification or polymer blending. For instance, blends of PHBHHx with poly(ethylene glycol) (PEG) [4] and poly(lactide) (PLA) [5] have been reported, and the physical properties of the blends could be tailored by modifying the blend composition, leading to good materials for in vivo applications. 
Electrospinning of polyesters is a flexible, easy, and cheap method for fabricating fiber mats for biomedical applications. In particular, it presents several advantages when developing scaffolds for tissue engineering applications [6]: similarity to the native extracellular matrix, control of porosity by adjusting the fiber size and not need of high temperatures. Besides, the parameters of the electrospinning process can be adjusted to finely tune to the characteristics of specific tissues. Recently, several companies have developed new methods derived from conventional electrospinning that make large scale production feasible, and have already been applied for many biodegradable polymers such as collagen, poly(L-lactide) (PLLA), poly( $\varepsilon$-caprolactone) (PCL), chitosan (CS), poly (lactide-co-glycolide) (PLGA), etc [7-9].

PCL is a biodegradable and biocompatible polyester with good blend compatibility widely applied in biomedical applications. However, PCL is expensive and has long biodegradability cycles, hence it can be blended with cheaper biodegradable polymers like CS, a polysaccharide composed mainly of $\beta$ - $(1,4)$-linked 2-deoxy-2-amino-D-glucopyranose units. It is an antibacterial, biocompatible biopolymer with good mechanical properties, widely used as wound dressing, drug delivery vehicle, artificial skin and bone substitute. Covalent linkages between the two polymers provide a new hybrid, able to exhibit synergistic features and enhanced performance.

In a previous study, CS-grafted-PCL/PHBHHx fiber blends with different compositions were developed via a wet electrospinning process [10], and their morphology, crystallization and melting behaviour, hydrophilicity, water uptake, cytotoxicity, viscoelastic, tensile and antibacterial properties were investigated. The present work focuses on the tribological performance of these novel biomaterials and its correlation with the morphology and the mechanical properties. Further, the biodegradability of the fibers has been analyzed.

\section{EXPERIMENTAL}

\subsection{Materials}

PHBHHx (HHx content of $12 \mathrm{~mol} \%, \mathrm{M}_{\mathrm{n}}=440000 \mathrm{~g} / \mathrm{mol} ; \mathrm{T}_{\mathrm{g}} \sim 2^{\circ} \mathrm{C}, \mathrm{T}_{\mathrm{m}} \sim 110^{\circ} \mathrm{C}, \mathrm{d}_{25^{\circ} \mathrm{C}}=$ $1.25 \mathrm{~g} / \mathrm{cm}^{3}$ ) was purchased from the Procter and Gamble Company (USA). Hydroxylterminated poly( $\varepsilon$-caprolactone), PCL $\left(\mathrm{Mn}=14000 \mathrm{~g} / \mathrm{mol} ; \mathrm{T}_{\mathrm{g}}-60^{\circ} \mathrm{C}, \mathrm{T}_{\mathrm{m}} \sim 60^{\circ} \mathrm{C}, \mathrm{d}_{25^{\circ} \mathrm{C}}=\right.$ $1.15 \mathrm{~g} / \mathrm{cm}^{3}$; PDI $=1.20$ ) was purchased from Polymer Source Inc. (Canada). Chitosan, CS $\left(\mathrm{M}_{\mathrm{w}}=60000-100000 \mathrm{Da}\right.$ and degree of deacetylation of 85\%) hexamethylene diisocyanate (HDI), stannous octoate $\left(\mathrm{Sn}[\mathrm{Oct}]_{2}\right), \mathrm{CHCl}_{3}$, trifluoroacetic acid (TFA), dichloromethane (DCM), phthalic anhydride, hydrazine monohydrate (98\%) and dimethylformamide (DMF), were purchased from Sigma-Aldrich.

\subsection{Synthesis of chitosan-grafted polycaprolactone}

CS-g-PCL was synthesized following a procedure adapted from Liu et al. [11] Briefly, CS was heated with excess phthalic anhydride in dried DMF under an inert atmosphere, precipitated and washed in ethanol to yield $N$-phthaloylchitosan (PHCS). Separately, a PCL solution in DMF was added to a flask containing HDI and $\mathrm{Sn}[\mathrm{Oct}]_{2}$ as a catalyst and heated at $70^{\circ} \mathrm{C}$ for $24 \mathrm{~h}$ under mechanical stirring and a nitrogen atmosphere to yield HDI-terminated PCL. Then, PHCS was dissolved in DMF and the HDI-terminated PCL was added (PHCS/PCL weight ratio $=1: 5)$. After $4 \mathrm{~h}$ at $80^{\circ} \mathrm{C}$ the resulting product $(\mathrm{PHCS}-\mathrm{g}-\mathrm{PCL}$ ) was separated by 
filtration and purified. Finally, PHCS-g-PCL was stirred in DMF at $100^{\circ} \mathrm{C}$ followed by addition of excess of hydrazine monohydrate, and the precipitate was washed with ethanol and dried in a vacuum oven. The percentage of grafting was $35.1 \%$ according to ${ }^{1} \mathrm{H}$ NMR spectroscopy.

\subsection{Preparation of CS-g-PCL/PHBHHx fibers}

The fibers were synthesized through a wet electrospinning process [10]. In short, CS-g-PCL/ PHBHHx blend solutions were prepared by dissolving the components in a mixture of TFA/ $\operatorname{DCM}(90: 10$ vol.\%) and stirred overnight on a rotary mixer. A syringe located on an advancement pump (speed $1.0 \mathrm{~mL} / \mathrm{h}$ ) was filled with each solution and connected to a high voltage power supply via a precision needle. A metallic plate was placed at the bottom of a methanol bath at $15 \mathrm{~cm}$ from the needle tip, and a voltage of $\sim 18 \mathrm{kV}$ was applied between the syringe tip and the metallic plate. The solution was then precipitated in the methanol bath, neutralized with $1 \mathrm{M} \mathrm{NaOH}$, washed with distilled water and methanol and dried under vacuum. The fiber blends are designated as 100/0,70/30, 50/50,30/70 and 0/100, where the numbers indicate the weight percentages of CS-g-PCL and PHBHHx, respectively.

\subsection{Characterization techniques}

The morphology was analyzed via scanning electron microscopy (SEM) carried out on a Philips XL30 Environmental SEM. An AutoPore III mercury porosimeter was used to determine the porosity. The degree of crystallinity was calculated from the DSC thermograms [10]. Tensile tests were performed on a 4204 mechanical tester at $23 \pm 2^{\circ} \mathrm{C}$ and $50 \pm 5 \% \mathrm{RH}$, according to the UNE-EN ISO 527-1 standard. Results are mean values of 6 measurements. Tribological properties were measured with a Microtest MT 400-98 equipment according to the ASTM D-1894- 11 standard at $23 \pm 2^{\circ} \mathrm{C}$ and $50 \pm 5 \% \mathrm{RH}$, with a testing speed of $0.25 \mathrm{~m} / \mathrm{s}$ and $1 \mathrm{~N}$ load. Five measurements were taken for each sample, and the mean values of friction coefficient and wear rate are reported. Hydrolytic degradation was analyzed by immersing the samples in phosphate buffered saline (PBS) at $\mathrm{pH} 7.4$ and $37^{\circ} \mathrm{C}$ for 7 weeks. The weight loss was calculated considering the mass before and after PBS incubation.

\section{RESULTS AND DISCUSSION}

\subsection{Porosity/Morphology of the fiber blends}

The level of porosity and pore size are key factors influencing cell attachment and proliferation of scaffolds for tissue-engineering. Pore sizes between 20 and $100 \mu \mathrm{m}$ are typically desirable for optimal cellular activity and viability. The porosity and pore size distribution of the fibers was measured by mercury intrusion porosimetry. All the samples have pores in the range of $2-50 \mu \mathrm{m}$, with main size values of $\sim 2$ and $20 \mu \mathrm{m}$, and a degree of porosity between 82 and $91 \%$, Fig. 1, typical of electrospun fiber mats. The porosity increases with increasing CS-g-PCL content, attributed to the higher viscosity of PCL than PHBHHx; in fact, the higher the viscosity, the more difficult to fill the pores during solvent evaporation, leading to a more porous fiber.

The inset of Fig. 1 is a SEM micrograph $(\times 400)$ of the 50/50 blend. Analogous morphology was found for the other fibers, with a mesh-like structure and a random fiber orientation. The 


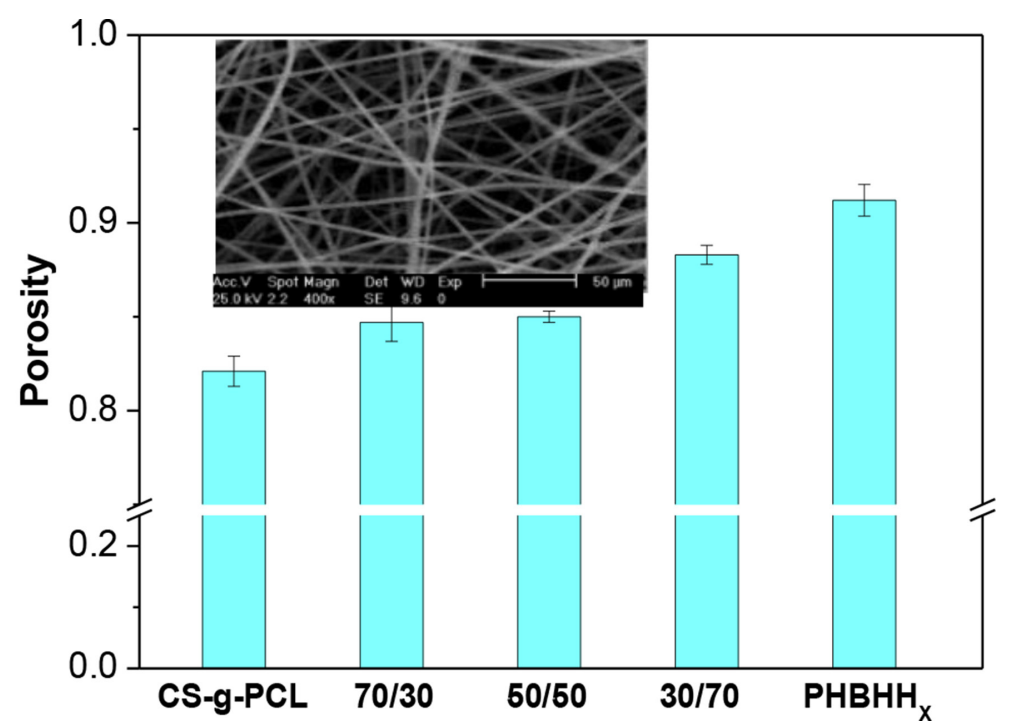

Figure 1: Porosity of CS-g-PCL/PHBHHx fiber blends. The inset shows a low magnification SEM micrograph of the 50/50 blend.

meshes are composed of tubular fibers with diameters in the range of $0.5-10 \mu \mathrm{m}$ and a smooth surface topography. All the fibers have average fiber diameters in the range of 4.8-5.6 $\mu \mathrm{m}$; as the PHBHHx content raises, the mean diameter somewhat increases.

CS-g-PCL/PHBHHx blends are immiscible at all compositions studied. SEM images at higher magnification $(\times 5000)$ revealed the different morphology of the samples. The grain sizes of CS-g-PCL and the 70/30 blend (Fig. 2a) were significantly bigger compared to neat PHBHHx and the 30/70 blend (Fig. 2c), which have a grainy appearance, with many small pores $(<300 \mathrm{~nm})$, whereas CS-g-PCL and the 70/30 blend have less and bigger pores and cobblestone appearance.

The overall level of crystallinity $\left(\mathrm{X}_{\mathrm{c}, \mathrm{T}}\right)$ of the blends was calculated from DSC thermograms [10], and is summarized in Table 1. CS-g-PCL shows the highest crystallinity, whereas PHBHHx the lowest, consistent with the amorphous character of the HHx monomers. $\mathrm{X}_{\mathrm{c}, \mathrm{T}}$ increases with increasing CS-g-PCL fraction, although it does not follow a linear trend as expected considering the rule of mixtures. Thus, the crystallinity of the 70/30 blend is comparable to that of CS-g-PCL, suggesting that the crystal structure could differ from that of typical PCL crystals, as reported for CS/PCL mixtures [6].

\subsection{Mechanical properties}

The mechanical properties of the developed fibers were measured via tensile tests at $23^{\circ} \mathrm{C}$, and the results are summarized in Table 1. The blends display Young's modulus (E) values intermediate between those of CS-g-PCL and PHBHHx, although they do not correlate with the sample composition but with the level of crystallinity: the higher the crystallinity, the higher the modulus. Accordingly, the 70/30 blend has a modulus comparable to that of CS-gPCL, while that of the 30/70 sample is only a little higher than that of PHBHHx. An analogous trend is found for the tensile strength $\left(\sigma_{\mathrm{y}}\right)$, apart from the fact that the value of the $30 / 70$ mixture is even lower than that of bare PHBHHx. This unexpected behaviour could be related 

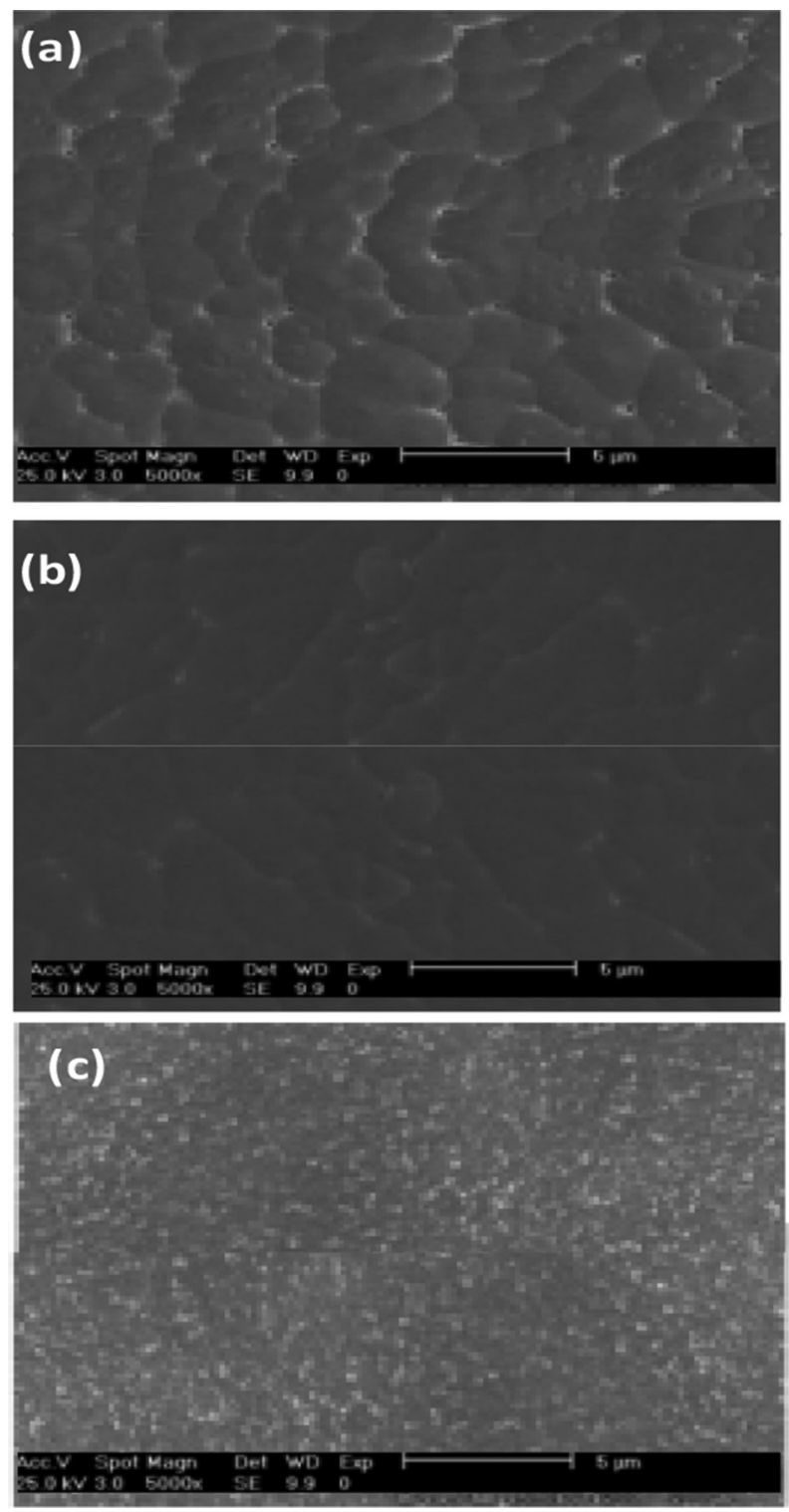

Figure 2: SEM images of CS-g-PCL/PHBHHx fiber blends: (a): 70/30; (b) 50/50; (c) 30/70.

to the morphology of this sample, since it presents many small pores, which are known to cause a worsening in mechanical properties [12]. Regarding the elongation at break $\left(\varepsilon_{\mathrm{b}}\right)$, the $70 / 30$ blend shows higher value than CS-g-PCL, whereas the 30/70 mixture lower value than the neat copolyester. The former blend should develop lamellar structures of crystalline material separated by softer amorphous parts that can fold into the "chevron" morphology [13], leading to a big plastic deformation, whilst the 30/70 blend would form individual large spherulites that provoke the lower ductility. Analogous trend is found for the toughness measured as the area under the tensile curve. The 70/30 blend displays better toughness than 
Table 1: Crystallinity and mechanical properties of the fiber blends. $X_{c, T}$ : overall degree of crystallinity E: Young's modulus; $\sigma_{\mathrm{y}}$ : tensile strength; $\varepsilon_{\mathrm{b}}$ : elongation at break; T: toughness.

\begin{tabular}{cccccc}
\hline & $\mathrm{E}$ & $\sigma_{\mathrm{y}}$ & $\varepsilon_{\mathrm{b}}$ & $\mathrm{T}$ & $\mathrm{X}_{\mathrm{c}, \mathrm{T}}$ \\
CS-g-PCL/PHBHHx & $(\mathrm{MPa})$ & $(\mathrm{MPa})$ & $(\%)$ & $\left(\mathrm{KJ} / \mathrm{m}^{2}\right)$ & $(\%)$ \\
\hline $100 / 0$ & 700 & 25 & 169 & 10.8 & 46.3 \\
$70 / 30$ & 624 & 26 & 185 & 12.5 & 43.8 \\
$50 / 50$ & 390 & 19 & 148 & 6.7 & 32.4 \\
$30 / 70$ & 242 & 9 & 96 & 2.4 & 26.2 \\
$0 / 100$ & 220 & 10 & 102 & 2.9 & 14.9 \\
\hline
\end{tabular}

CS-g-PCL due to the combination of improved ductility and strength, whereas the 30/70 has a lower value than PHBHHx due to its lower ductility. Further, the grain sizes of CS-g-PCL and the 70/30 blend are larger than those of PHBHHx and the 30/70 blend, and big grains typically lead to improved impact strength [12]. In summary, results reveal that an optimal combination of stiffness, strength, ductility and toughness is attained at a particular blend composition (70/30), likely due to a synergistic effect between the components. Synergistic effects in ductility and impact strength have also been reported for a 3:1 poliglecaprone (PGC)/PCL fibrous blend [14] as well as for a 85/15 PLA/PCL mixture compatibilized with lysine triisocyanate [15].

\subsection{Tribological properties}

Electrospun fibers are usually fragile and susceptible to wear and delamination even under conditions of tender handling, which limits their service lifetime. To analyze this behaviour, the coefficient of friction $(\mu)$ and specific wear rate $\left(W_{s p}\right)$ were measured, and the results are plotted in Fig. 3. A low coefficient of friction is desirable since it implies that the fiber experiences small forces during abrasive contact with the counterface, which would lead to low wear.

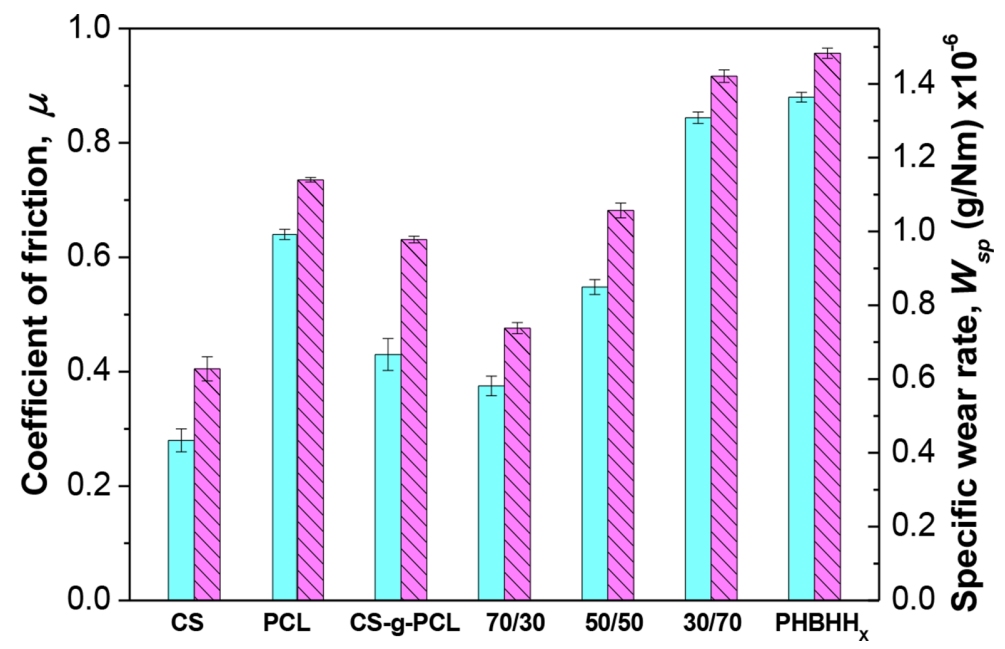

Figure 3: Coefficient of friction (solid bars) and specific wear rate (dashed bars) of the fibers. 
$\mu$ of CS-g-PCL is around 0.43 , intermediate between the values of neat CS and PCL, while that of PHBHHx is around 0.87 . The blends show $\mu$ values in the range of $0.37-0.85$, although these do not directly correlate with the sample composition but with the tensile properties of the fiber (i.e. modulus, strength): the higher E, the lower $\mu$. Thus, the stiffest sample, the $70 / 30$ mixture, presents the smallest $\mu$ value while PHBHHx and the 30/70 mixture, the softest ones, display the highest. A similar trend is found for the wear rate: there is about 2-fold reduction in the specific wear rate of the 70/30 mixture compared to neat PBHHHx. The observed decrement likely has also a strong correlation with the morphology of the samples: PHBHHx and the 30/70 blend have a grainy appearance, with many small pores, whilst CSg-PCL and the 70/30 blend exhibit fewer and larger pores, and reduction in porosity is advantageous for improving tribological properties [16] since the debris accumulates into the pore spaces within the fiber mat, consequently roughening the surface and increasing the coefficient of friction, and in turn, leading to abrasive wear.

The wear can be due to different mechanisms, namely physicochemical interactions, roughness interactions or macroscopic deformation [17]. Apart from the morphological factors discussed above and the influence of the chemical composition, the abrasive wear for these nanofiber mats could arise from macroscopic deformation induced when going beyond the yield stress of the fibers. Thus, for polymeric-based materials, the wear properties directly correlate with the mechanical performance; in particular, deformation of a surface depends on the indentation hardness, the frictional force and the material removal. These three parameters can be correlated through the Ratner-Lancaster equation [18]. In the case of nanofiber mats, a modified version of the Ratner-Lancaster relationship has been proposed [19]: $W_{s p} \approx \mathrm{C}(\rho \mu \mathrm{L}) /\left(\sigma_{\mathrm{y}} \varepsilon_{\mathrm{y}}\right)$ in which $\mathrm{C}$ is a constant, $\rho$ the density, $\mathrm{L}$ the applied load, $\sigma_{\mathrm{y}}$ the tensile strength and $\varepsilon_{\mathrm{y}}$ the yield strain. Figure 4 plots the specific wear rate of the nanofiber mats for $1 \mathrm{~N}$ applied load vs. $\left(\sigma_{\mathrm{y}} \varepsilon_{\mathrm{y}}\right)^{-1}$. A perfectly linear relationship is found, following the modified Ratner-Lancaster equation, which corroborates that there is a direct correlation between the wear rate and the mechanical properties, pointing that the mechanism of wear is mainly due to the breakage of fibers that is also responsible for yield in these fiber mats.

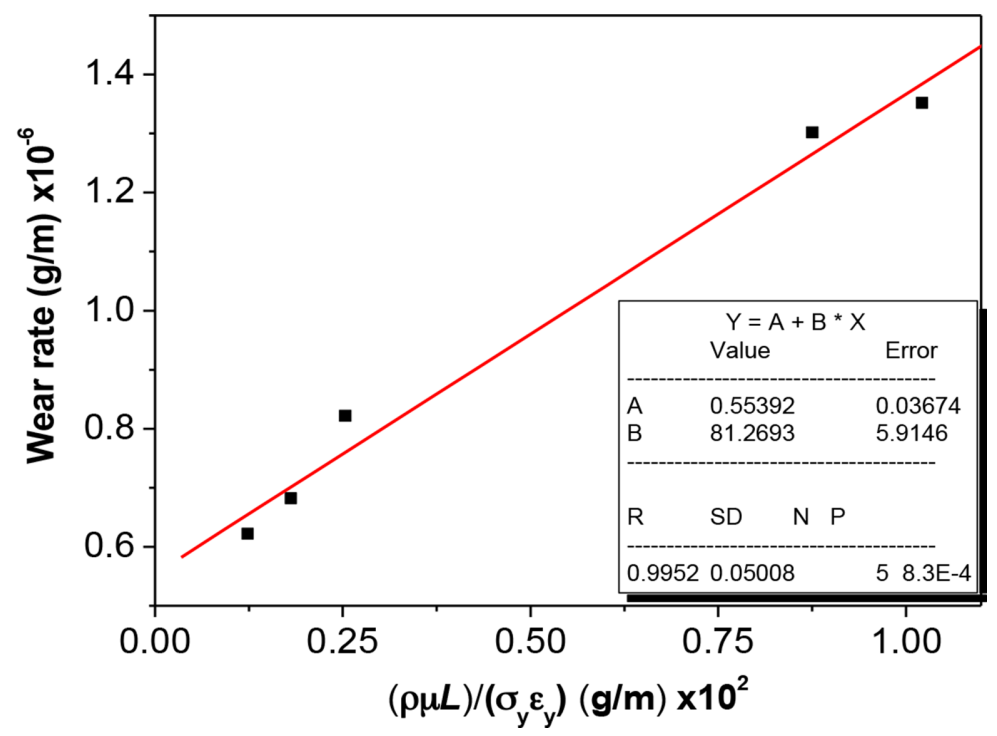

Figure 4: Wear rate vs. $\rho \mu \mathrm{L} /\left(\sigma_{\mathrm{y}} \varepsilon_{\mathrm{y})}\right.$ for $1 \mathrm{~N}$ applied load. 


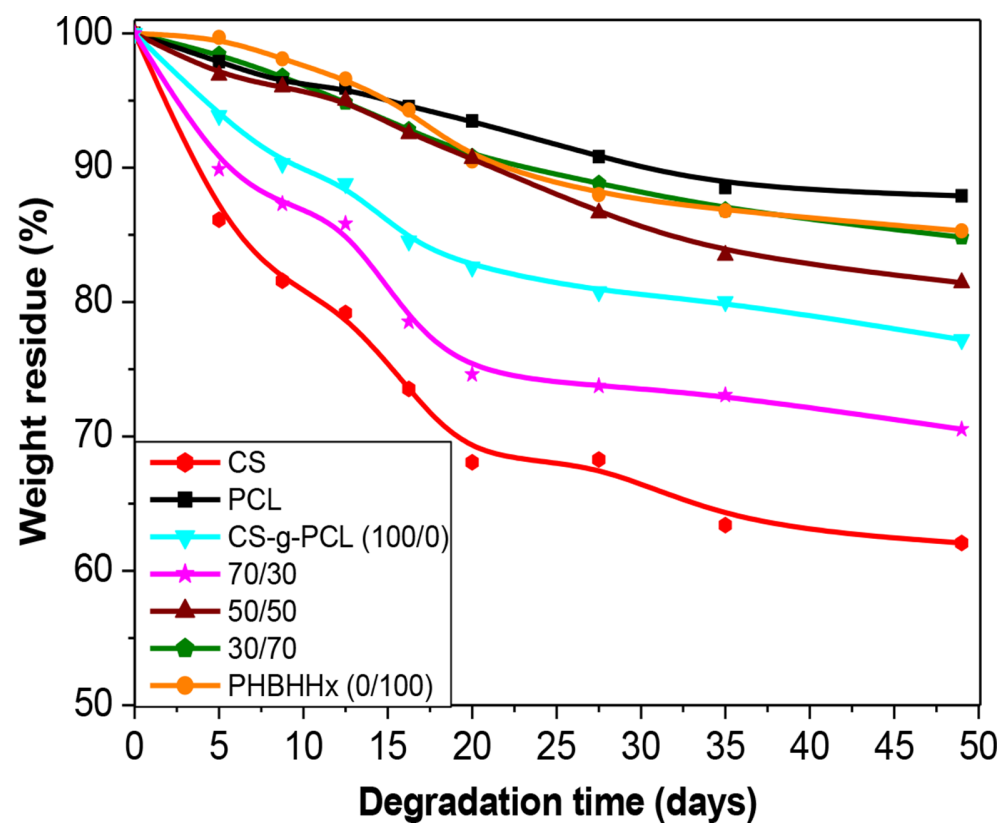

Figure 5: Percentage weight residue of the different fibers as a function of time.

\subsection{Biodegradability}

A key parameter for the selection of materials for tissue engineering applications is the biodegradability. For that reason, an in vitro degradation study in PBS at $37^{\circ} \mathrm{C}$ was carried out, and the percentage weight loss of the different fibers as a function of time is plotted in Fig. 5. The degradation rate is influenced by many parameters, namely the molecular weight, degree of crystallinity, spherulite size and level of porosity. PCL exhibits a weight loss of about $12 \%$ after 7 weeks, attributed to hydrolytic degradation of aliphatic ester linkages, a process that starts with a water uptake phase followed by random hydrolytic scission of the ester bonds [20]. Considerably higher weight loss is detected for neat CS $(\sim 38 \%)$, ascribed to its higher water uptake [10], whilst CS-g-PCL shows an intermediate value of about $23 \%$. Conversely, neat PHBHHx only loses around $15 \%$ weight, and the degradation has been reported to occur preferentially in the amorphous regions [21]. The weight loss of the blends decreases with increasing PHBHHx content, following a similar trend to the water uptake [10]. Nonetheless, the 70/30 blend loses less weight than the expected according to its composition, probably related to its high level of crystallinity, since the crystalline domains are less permeable to water, slowing down the hydrolysis. This slower degradation is advantageous from a practical point of view.

\section{CONCLUSIONS}

CS-g-PCL/PHBHHx fiber blends in different compositions have been developed by means of a wet electrospinning process, and their morphology, biodegradability, mechanical and tribological properties have been analyzed. The porosity increased with increasing CS-gPCL content, and the blends exhibited distinct morphology: CS-g-PCL and the 70/30 blend had cobblestone appearance, while PHBHHx and the 30/70 blend had a grainy morphology. The grain sizes of CS-g-PCL and the 70/30 blend were larger than those of PHBHHx 
and the 30/70 blend. The crystallinity also increased with increasing CS-g-PCL fraction, although it does not follow a linear trend as expected considering the rule of mixtures. The Young's modulus, tensile strength and elongation at break decreased with increasing PHBHHx loading. A direct correlation was found between the tribological and the mechanical properties. The specific wear rate of the nanofiber mats can be described by a modified Ratner-Lancaster relationship for wear rate of polymeric materials, suggesting that the mechanism of wear is principally due to the breakage of fibers that is also responsible for yield. Altogether, experimental results reveal the existence of a particular blend composition $(70 / 30)$ at which the interactions between the components lead to synergistic effects, resulting in an optimum combination of superior stiffness, strength, ductility and toughness and reduced coefficient of friction and wear rate; this unexpected behaviour is attributed to the lower porosity and higher level of crystallinity of this sample. Besides, it has the slowest degradation rate, which is advantageous from a practical viewpoint. The results obtained in this study demonstrate the immense potential of these novel fiber blends, particularly that with a composition of 70/30, to be used in biomedical applications, especially for tissue engineering.

\section{ACKNOWLEDGEMENT}

Dr. Ana Díez-Pascual wishes to acknowledge the Ministerio de Economía y Competitividad (MINECO) for a "Ramón y Cajal" Senior Research Fellowship cofinanced by the EU and for financial support (project ref. CTQ2015-66575-P).

\section{REFERENCES}

[1] Laycock, B., Halley, P., Pratt, S., Werker, A. \& Lant, P., The chemomechanical properties of microbial polyhydroxyalkanoates. Progress Polymer Science, 38, pp. 536-583, 2013. https://doi.org/10.1016/j.progpolymsci.2012.06.003

[2] Mekonnen, T., Mussone, P., Khalil, H. \& Bressler, D., Progress in bio-based plastics and plasticizing modifications. Journal of Materials Chemistry A, 1, pp. 13379-13398, 2013. https://doi.org/10.1039/c3ta12555f

[3] Chang, H.M., Wang, Z.H., Luo, H.N., Xu, M., Ren, X.Y., Zheng, G.X., Wu, B.J., Zhang, X.H., Lu, X.Y., Chen, F., Jing, X.H. \& Wang, L., Poly(3-hydroxybutyrate-co-3hydroxyhexanoate)-based scaffolds for tissue engineering. Brazilian Journal of Medical and Biological Research, 47, pp. 533-539, 2014. https://doi.org/10.1590/1414-431X20143930

[4] Qu, X.H., Wu, Q., Zhang, K.Y. \& Chen, G.Q., In vivo studies of poly(3-hydroxybutyrate-co-3-hydroxyhexanoate) based polymers: biodegradation and tissue reactions. Biomaterials, 27, pp. 3540-3548, 2006. https://doi.org/10.1016/j.biomaterials.2006.02.015

[5] Zhao, Q., Wang, S., Kong, M., Geng, W., Li, R.K., Song, C. \& Kong, D., Phase morphology, physical properties, and biodegradation behavior of novel PLA/PHBHHx blends. Journal Biomedical Materials Research Part B: Applied Biomaterials, 100, pp. 23-31, 2012. https://doi.org/10.1002/jbm.b.31915

[6] Ladd, M.R., Hill, T.K., Yoo, J.J. \& Lee, S.J., Electrospun Nanofibers in Tissue Engineering, InTechOpen: Rijeka, 2011. 
[7] Zong, X., Kim, K.S., Fang, D., Ran, S., Hsiaoa, B.S. \& Chu, B., Structure and process relationship of electrospun bioabsorbable nanofiber membranes. Polymer, 43, pp. 4403-4412, 2002. https://doi.org/10.1016/S0032-3861(02)00275-6

[8] Reneker, D.H., Kataphinan, W., Theron, A., Zussman, E. \& Yarin, A. L. Nanofiber garlands of polycaprolactone by electrospinning. Polymer, 43, pp. 6785-6794, 2002. https://doi.org/10.1016/S0032-3861(02)00595-5

[9] Zong, X., Ran, S., Kim, K.S., Fang, D., Hsiao, B.S. \& Chu, B., Structure and morphology changes during in vitro degradation of electrospun poly(glicolide-co-lactide) nanofiber membrane. Biomacromolecules, 4, pp. 416-423, 2003. https://doi.org/10.1021/bm025717o

[10] Diez-Pascual, A.M. \& Diez-Vicente, A.L., Electrospun fibers of chitosan-grafted polycaprolactone/poly(3-hydroxybutyrate-co-3-hydroxyhexanoate) blends. Journal of Materials Chemistry B, 4, pp. 600-612, 2016. https://doi.org/10.1039/C5TB01861G

[11] Liu, L., Li, Y., Liu, H. \& Fang, Y., Synthesis and characterization of chitosan-graftpolycaprolactone copolymers. European Polymer Journal, 40, pp. 2739-2744, 2004. https://doi.org/10.1016/j.eurpolymj.2004.07.016

[12] Callister, W.D., Materials Science and Engineering: An introduction, John Wiley \& Sons, Inc.: New York, 2007.

[13] Michlera, G.H. \& Baltá-Calleja, F.J., Mechanical Properties of Polymers based on Nanostructure and Morphology, CRC press: Florida, p. 407, 2005. https://doi.org/10.1201/9781420027136

[14] Patel, H. N. Fibro-porous poliglecaprone/polycaprolactone conduits: synergistic effect of composition and in vitro degradation on mechanical properties. Polymer International, 64, pp. 547-555, 2015. https://doi.org/10.1002/pi.4834

[15] Takayama, T. \& Todo, M., Improvement of fracture properties of PLA/PCL polymer due to LTI addition. Journal of Materials Science, 41, pp. 4989-4992, 2006. https://doi.org/10.1007/s10853-006-0137-1

[16] Shi, S.F.E.L., Guo, Z.G. \& Liu, W.M., The recent progress of tribological biomaterials. Biosurface and Tribology, 1, pp. 81-97, 2015. https://doi.org/10.1016/j.bsbt.2015.06.002

[17] Suh, N.P., Tribophysics, Prentice-Hall: New Jersey, 1986.

[18] Lancaster, J.K., Abrasive wear of polymers. Wear, 14, pp. 223-239, 1969. https://doi.org/10.1016/0043-1648(69)90047-7

[19] Mannarino, M.M. \& Rutledge, G.C., Mechanical and tribological properties of electrospun PA 6(3)T fiber mats. Polymer, 14, pp. 3017-3025, 2012. https://doi.org/10.1016/j.polymer.2012.04.039

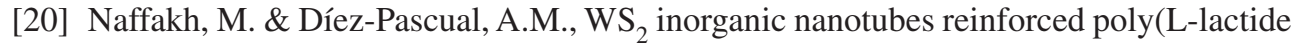
acide)/hydroxyapatite hybrid composite biomateriales. RSC Advances, 5, pp. 6551465525, 2015.

https://doi.org/10.1039/C5RA10707E

[21] Wang, Y., Mo, W., Yao, H., Wu, Q., Chen, J. \& Chen, G., Biodegradation studies of poly(3-hydroxybutyrate-co-3-hydroxyhexanoate). Polymer Degradation Stability, 85, pp. 815-821, 2004.

https://doi.org/10.1016/j.polymdegradstab.2004.02.010 